\title{
Disruption of Right Prefrontal Cortex by Low-Frequency Repetitive Transcranial Magnetic Stimulation Induces Risk-Taking Behavior
}

\author{
Daria Knoch, ${ }^{1}$ Lorena R. R. Gianotti, ${ }^{3}$ Alvaro Pascual-Leone, ${ }^{4}$ Valerie Treyer, ${ }^{2}$ Marianne Regard, ${ }^{1}$ Martin Hohmann, ${ }^{1}$ \\ and Peter Brugger ${ }^{1}$ \\ ${ }^{1}$ Department of Neurology, ${ }^{2}$ PET Center, Division of Nuclear Medicine, University Hospital Zurich, 8091 Zurich, Switzerland, ${ }^{3}$ The KEY Institute for Brain- \\ Mind Research, University Hospital of Psychiatry, 8032 Zurich, Switzerland, and ${ }^{4}$ Center for Noninvasive Brain Stimulation, Harvard Medical School and \\ Beth Israel Deaconess Medical Center, Boston, Massachusetts 02215
}

\begin{abstract}
Decisions require careful weighing of the risks and benefits associated with a choice. Some people need to be offered large rewards to balance even minimal risks, whereas others take great risks in the hope for an only minimal benefit. We show here that risk-taking is a modifiable behavior that depends on right hemisphere prefrontal activity. We used low-frequency, repetitive transcranial magnetic stimulation to transiently disrupt left or right dorsolateral prefrontal cortex (DLPFC) function before applying a well known gambling paradigm that provides a measure of decision-making under risk. Individuals displayed significantly riskier decision-making after disruption of the right, but not the left, DLPFC. Our findings suggest that the right DLPFC plays a crucial role in the suppression of superficially seductive options. This confirms the asymmetric role of the prefrontal cortex in decision-making and reveals that this fundamental human capacity can be manipulated in normal subjects through cortical stimulation. The ability to modify risk-taking behavior may be translated into therapeutic interventions for disorders such as drug abuse or pathological gambling.
\end{abstract}

Key words: decision-making; dorsolateral prefrontal cortex; inhibitory control; laterality; transcranial magnetic stimulation; risk-taking

\section{Introduction}

The ability to make correct decisions in a complex and changing environment requires careful weighing of risks and benefits. The prefrontal cortex (PFC) appears to be critical in such decisionmaking processes. Decision-making behavior in adolescents, generally more risk-taking in nature, is thought to be the manifestation of an immature prefrontal cortex (Chambers et al., 2003), and patients with traumatic brain injuries or other pathologies affecting the PFC show a tendency for riskier, "out-ofcharacter" decision-making and an apparent disregard for negative consequences of their actions (Bechara et al., 1996; Rahman et al., 2001). This seems particularly true for patients with rightsided lesions (Tranel et al., 2002; Clark et al., 2003), although still little is known about the lateralization of the neural mechanisms involved in decision-making. Some functional imaging studies suggest that the right prefrontal cortex may be particularly critical for the regulation of risk-taking behavior (Rogers et al., 1999; Ernst et al., 2002; Fishbein et al., 2005). However, these studies do not provide a direct causal link between structure and function. We used repetitive transcranial magnetic stimulation (rTMS) to

Received Feb. 22, 2006; revised April 6, 2006; accepted May 9, 2006

This work was supported by the Swiss National Science Foundation and UBS AG. We thank E. Wintsch for programming the risk task used in this study.

Correspondence should be addressed to Dr. Daria Knoch, Department of Neurology, University Hospital Zurich, Frauenklinikstrasse 26, CH-8091 Zurich, Switzerland. E-mail: daria.knoch@usz.ch.

DOI:10.1523/JNEUROSCI.0804-06.2006

Copyright $\odot 2006$ Society for Neuroscience $\quad$ 0270-6474/06/266469-04\$15.00/0 transiently disrupt left or right prefrontal function and thus examine whether risk-taking strategies can be modified in healthy individuals and provide direct evidence for the causal role of lateralized hemispheric control of risk-taking.

\section{Materials and Methods}

Subjects. We studied 27 right-handed men (mean age, 23.8 years; range, 21-31 years). All provided written informed consent to participate in the study that had been approved by the local ethics committee. All were naive to TMS and had no history of psychiatric illness or neurological disorders. Subjects received $80 \mathrm{CHF}$ (Swiss francs) for their participation. They were randomly assigned to either left or right prefrontal rTMS or sham stimulation. There was no difference between groups with respect to age $\left(F_{(2,24)}=1.64 ; p=0.215\right)$. No subject reported any adverse side effects concerning pain on the scalp or headaches after the experiment.

Risk task. Subjects were randomly assigned to receive sham or verum rTMS to the left or the right dorsolateral PFC (DLPFC) before performing the Risk Task (Rogers et al., 1999), a well known gambling paradigm that provides a measure of decision-making under risk with little requirements on strategy and working memory. In each of the 100 trials, subjects were presented with six horizontally arranged boxes that could be pink or blue (Fig. 1). The ratio of pink and blue boxes varied from trial to trial and could be 5:1, 4:2, or 3:3. Subjects had to pick the color of the box that hid the "winning token." They were told that the token was equally likely to be hidden in any of the boxes. Therefore, for each trial, the ratio of pink to blue boxes (referred to as "level of risk") effectively determined the probability of finding that winning token and thus the level of risk of the choice. Subjects were rewarded with points for picking the color of the box hiding the winning token and punished by loosing points for picking the incorrect color. The amount of reward (or penalty) points 




Figure 1. Experimental design. Subjects received $1 \mathrm{~Hz}$ rTMS during $15 \mathrm{~min}(\boldsymbol{b})$. The positioning of the coil over the left or right DLPFC was confirmed for all subjects using a threedimensional MRI sequence with vitamin E capsule in place (sample shows right DLPFC). Four trials of the Risk Task are shown in $\boldsymbol{d}$. Subjects performed all 100 trials in $\sim 7 \mathrm{~min}$. The ratio of pink and blue boxes changed from trial to trial, and this is referred to in the text as level of risk. The numbers inside the bottom two boxes depict the reward/punishment sizes associated with the respective colors, in the text referred to as balance of reward. Reward associated with either choice of box color was fixed ( 10 vs 90,20 vs 80,30 vs 70 , and 40 vs 60 ); the larger reward (and penalty) was associated with choice of the high-risk prospect, whereas the smallest reward (and penalty) was associated with the choice of the low-risk prospect, providing inherent reward conflict typical of risk-taking situations. The subject's task was to select one of these boxes to indicate the color of the box thought to hide a winning token (not shown in the samples). Subjects had to rate their mood before $(\boldsymbol{a})$ and immediately after $(\boldsymbol{c})$ the rTMS train.

associated with any one scenario (for example, five blue and one pink box) varied (90:10, 80:20, 70:30, 60:40; referred to as "balance of reward") and was indicated in the bottom two boxes on the screen (Fig. 1). The larger reward (and penalty) was always associated with choice of the high-risk prospect (i.e., the lower likelihood to obtain the winning outcome, which was the color with the fewest number of boxes), whereas the smallest reward (and penalty) was associated with choice of the low-risk prospect. Thus, in a trial with five blue boxes and one pink box (supplemental Fig. 1, available at www.jneurosci.org as supplemental material), the winning token was much more likely to be hidden in a blue box ( 5 in 6 probability) than in the lone pink box ( 1 in 6 probability). In this case, if the subject picked pink and was correct, she would be awarded the number of points indicated in the lower pink box, which would always be greater than the points associated with the choice of blue. Conversely, picking pink and missing the winning token would result in a loss of the same, larger number of points. Subjects' aim was to earn as many points as possible.

Transcranial magnetic stimulation. rTMS was administered to the DLPFC before subjects performed the task ("off-line paradigm") (Fig. 1) using a Magstim (Rapid Magnetic Stimulator; Magstim, Winchester, MA) and figure-of-eight coil (70-mm-diameter double circle, air cooled). The position of the DLPFC was defined as $6 \mathrm{~cm}$ anterior to the motor cortex, at the same lateral distance along the convexity from the midsagittal plane as the hand motor representation. A T1-weighted magnetic resonance imaging (MRI) with vitamin $E$ capsules placed on the previously marked TMS target positions (Fig. 1) was acquired for all subjects who received verum stimulation to confirm the proper positioning of the TMS coil. The coil was positioned tangentially to the scalp pointing in an anteromedial direction, $45^{\circ}$ from the midsagittal axis of the subject's head. Stimulation intensity was set at $100 \%$ of the individual resting motor threshold (MT), as determined following current guidelines. Groups of subjects did not differ with respect to $\mathrm{MT}\left(t_{(16)}=0.68\right.$; $p=0.634$ ). In the left hemisphere (right hand), the MT was $37-50 \%$ of maximal stimulator output, and, for the right hemisphere (left hand), the MT was 35-52\% of maximal stimulator output. Subjects received a single, $15 \mathrm{~min}, 1 \mathrm{~Hz}$ rTMS train (900 pulses) over the left DLPFC or right DLPFC or received sham stimulation (counterbalanced over the left and right DLPFC). These rTMS parameters are well within currently recommended guidelines (Wassermann, 1998) and result in a suppression of excitability of the targeted cortical region for several minutes after completion of the rTMS train (Robertson et al., 2003).

Statistical analysis. Principal measures of interest were the percentage of instances in which subjects chose the high-probability option (the color corresponding to more boxes) and the time it took the subjects to make the choice of a color. We refer to the first as "percentage choice of the low-risk prospect" and to the second as "decision times." Performance on the entire task of 100 trials with the exception of neutral risk scenarios (i.e., equal number of blue and pink boxes) was analyzed. For statistical analyses, the proportion of trials on which subjects chose the low-risk prospect was arcsine transformed, as is recommended whenever the variance is proportional to the mean (Howell, 1997). For simplicity, the data on percentage choice of the low-risk prospect shown in the figures represent untransformed values. The two principle measures (i.e., percentage choice of the low-risk prospect and decision times) were subjected to three-way repeated-measures ANOVA with group (right TMS, left TMS, sham) as between-subject factor and level of risk $(5: 1,4: 2)$ and balance of reward (90:10, 80:20, 70:30, 60:40) as within-subject factors. There was no time limit for subjects' responses, but trials with outlier reaction times, i.e., slower or faster than 2 SDs of the individual mean, were discarded from the analysis. Moreover, to test differences in decision time between "risky" and "safe" decisions, we computed a two-way ANOVA with group (right TMS, left TMS, sham) as between-subject factor and quality of decision (safe, risky) as within-subject factor. Five subjects (four left TMS, one sham) were not included in this analysis because they made no risky choices. To compare the effect of rTMS on mood, we conducted a two-way repeated-measures ANOVA with group (right TMS, left TMS, sham) as between-subject factor and test session (before TMS, after TMS) as within-subject factor. Differences between groups in the total time needed to complete the task (100 trials) and in the total score (100 trials) were tested with a one-way ANOVA with group (right TMS, left TMS, sham) as between-subject factor. When justified by the ANOVA analysis, significant effects were further analyzed by Scheffé's test. Differences were considered statistically significant at $p<0.05$.

\section{Results}

All subjects performed the task well, completing all trials in $\sim 7$ min regardless of TMS condition $\left(F_{(2,24)}=1.10 ; p=0.350\right)$ and showing the expected influence of risk and balance of reward on decision speed (supplemental Fig. 2, available at www.jneurosci. org as supplemental material). However, the number of points earned was significantly dependent on the stimulation condition (Fig. 2a). Subjects stimulated over the right DLPFC earned significantly less points and were more likely to choose the high-risk prospect than subjects in the other study groups (Fig. $2 b$ ). For all subjects, the percentage of conservative (safe) decisions increased significantly as the reward decreased for the high-risk prospect (Fig. 2c). This and the lack of significant interaction between group and balance of reward (Fig. $2 c$ ) reveals that the subjects' tendency to select riskier options after right DLPFC rTMS might be better interpreted as a relative insensitivity to risk than as a heightened sensitivity to reward.

\section{Discussion}

There are several reasons that could account for the disadvantageous, risky decision-making after right prefrontal stimulation. 
a

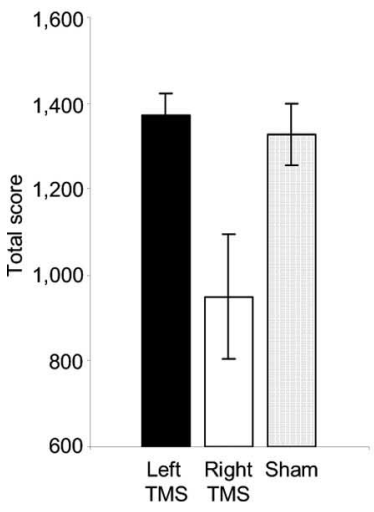

b

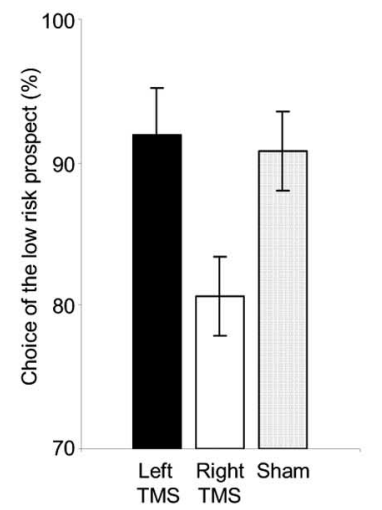

C

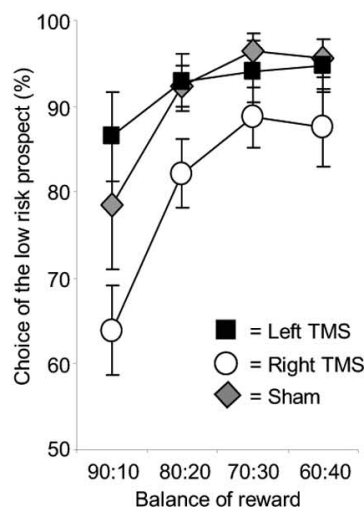

Figure 2. $\boldsymbol{a}$, Total points earned in 100 trials (means \pm SEM). One-way ANOVA revealed a significant main effect of group $\left(F_{(2,24)}=5.60 ; p=0.011\right)$. Subjects stimulated over the right DLPFC earned significantly fewer points than those who received $\operatorname{sham}(p=0.040)$ or the left DLPFC who received real rTMS $(p=0.020) \cdot \boldsymbol{b}$, Percentage choice of the low-risk prospect (means \pm SEM) for all three groups. Repeated-measures ANOVA of subject group $\times$ level of risk $\times$ balance of reward revealed a main effect of group $\left(F_{(2,24)}=4.92 ; p=0.016\right)$, and post hoc analysis demonstrated that subjects who received rTMS over the right DLPFC were more likely to choose the high-risk prospect than those stimulated over the left DLPFC ( $p=0.034)$ or those who received sham rTMS ( $p=0.048)$. Indeed, seven of nine subjects who received right DLPFC $r T M S$ ultimately performed among the subjects more prone to risk-taking, whereas most of those who received left DLPFC rTMS ended up in the group of subjects who were less inclined toward risk-taking. c, Percentage choice of the low-risk prospect (means \pm SEM) as a function of the balance of reward. Repeated-measures ANOVA of subject group $\times$ level of risk $\times$ balance of reward revealed a main effect of balance of reward $\left(F_{(3,72)}=17.19 ; p<0.001\right)$. No interaction between group and balance of reward was found $\left(F_{(6,72)}=1.03 ; p=0.414\right)$.

inhibitory control, leading to overly risky decision-making. A potential objection to any account in terms of inhibition is that orbital rather than dorsal areas of the PFC are traditionally implicated in inhibitory control functions. However, orbitofrontal and dorsolateral prefrontal structures are densely interconnected (Ghashghaei and Barbas, 2002), and stimulation of one leads to coactivations of the other (Li et al., 2004; Knoch et al., 2006). We hypothesize that DLPFC rTMS affected orbitofrontal cortex. and such distal impact accounts for the observed behavioral effects. Importantly, TMS over the right PFC did not influence decision times and certainly not in a way that could be interpreted as reflecting an especially hasty behavior. ANOVA of group $\times$ quality of decision (safe, risky) revealed no main effect for group $\left(F_{(2,19)}\right.$ $=0.44 ; p=0.651)$ but a main effect for quality of decision. Safe decisions were faster than risky decisions $\left(F_{(1,19)}=45.52\right.$; $p<0.001)$. Specifically, the observation that, in all groups, risky choices took longer than safe choices speaks against a disinhibition at motor level.

First, happy individuals tend to overestimate the likelihood of positive and underestimate the likelihood of negative outcomes of events (Johnson and Tversky, 1983). Therefore, the enhanced readiness to display risky behavior after right PFC rTMS could be attributable to an increase in happiness induced by rTMS (Gershon et al., 2003). However, we assessed subjects' mood before and after stimulation using visual analog scales and found them to be independent of the hemisphere stimulated. ANOVA of group $\times$ test session (before TMS, after TMS) revealed only a main effect for test session $\left(F_{(2,24)}=15.38 ; p<0.001\right)$. Stimulation over the left and right DLPFC significantly altered mood toward less "happy," but all three groups rated their mood as still positive.

Second, in accordance with the somatic marker hypothesis (Damasio, 1996; Bechara and Damasio, 2005), right PFC rTMS could have affected the detection or processing of marker signals that arise in bioregulatory processes and guide decision-making. The ventromedial PFC, particularly on the right (Critchley et al., 2000), is indeed believed to play a crucial role in the interpretation and regulation of such bodily sensations. However, the somatic marker hypothesis was formulated in the framework of experiments on the Iowa Gambling Task, which measures decision-making under ambiguity rather than risk. Decisionmaking under ambiguity does not allow for a fast cost-benefit analysis guided by simple rules. Consecutive outcomes can only be guessed by gradually learning the pattern of reward and punishment associated with specific options. In contrast, in the Risk Task, event probabilities are always explicitly given, learning is minimized, and guidance by any markers is likely less critical for effective performance.

We propose that what is primarily needed in the Risk Task is an active suppression of an option that appears most seductive because of the immediate higher payoffs. However, this inclination is balanced by control mechanisms sensitive to the negative consequences of high losses. Our results demonstrate that suppression of the right, but not the left, PFC by rTMS reduces
Therefore, we conclude that the right (not the left) PFC plays a crucial role in the suppressive control of superficially seductive options. This is consistent with findings of preferential righthemisphere involvement for inhibitory control of behavior in go/no-go paradigms (Garavan et al., 1999; Aron et al., 2003) and with clinical evidence suggesting a preferential right hemispheric lateralization of syndromes, such as drug abuse or nonsubstance addictions, in which impairments of decision-making seem to reflect a breakdown of these control processes (Starkstein and Robinson, 1997). Indeed, the demonstrated TMS-induced "appetite for risk" resembles that reported previously for drug abusers' behavior (Fishbein et al., 2005), which suggests a failure in self-regulation (Lieberman and Eisenberger, 2004) or a deficient reflective system (Bechara, 2005) or "cool" system (Metcalfe and Mischel, 1999). It is tempting to speculate whether highfrequency (i.e., 10-20 Hz), instead of low-frequency, rTMS over the right PFC would diminish, rather than increase, subjects' risk behavior. In a recent experiment (Knoch et al., 2005), we manipulated the naturally occurring inhibition of an automatized habit by rTMS over the PFC and either suppressed or released habitual responses depending on stimulation frequencies. Consistent with this notion, in cocaine addicts, pilot data suggest that targeting the right, but not the left, DLPFC with $20 \mathrm{~Hz}$ rTMS leads to a significant suppression of craving (A. J. Camprodon, J. MartinezRaga, and A. Pascual-Leone, unpublished observations). The observed laterality effect in our study is also compatible with the view that the right PFC is particularly sensitive to punishments or negative events (Davidson, 2004; Bechara and Damasio, 2005). On this account, inhibition of this area produced a selective neglect for negative consequences.

It is important to note that we provided our subjects with no monetary compensation for points accumulated during the task. Instead, subjects were paid a set compensation amount for their participation in the study. The fact that no monetary significance was attached to the points accumulated by the end of the task could be perceived as a limitation, given that there is a lack of 
incentive for subjects to do well during the game. However, most other studies using similar tasks also do not provide monetary rewards (Rogers et al., 1999; Manes et al., 2002; Clark et al., 2003; Fishbein et al., 2005). Given the findings of significant group performance differences in the hypothesized direction in this and other studies, this is likely not a cause for our results.

In summary, we show that risky decision-making engages a predominantly right-lateralized neural network, suggesting that adaptive decision-making depends on the degree of activation of right-sided prefrontal structures. In real-life scenarios, the substantial differences among individuals in risk proneness may correspond to different levels of activity in the right prefrontal cortex. The higher this level, the lower one's appetite for risk. If this turns out to be true, high-frequency rTMS could be used to increase activity of the right PFC in a therapeutic framework to enhance cognitive control and adaptive decision-making.

\section{References}

Aron AR, Fletcher PC, Bullmore ET, Sahakian BJ, Robbins TW (2003) Stopsignal inhibition disrupted by damage to right inferior frontal gyrus in humans. Nat Neurosci 6:115-116.

Bechara A (2005) Decision making, impulse control and loss of willpower to resist drugs: a neurocognitive perspective. Nat Neurosci 8:1458-1463.

Bechara A, Damasio AR (2005) The somatic marker hypothesis: a neural theory of economic decision. Games Econ Behav 52:336-372.

Bechara A, Tranel D, Damasio H, Damasio AR (1996) Failure to respond automatically to anticipated future outcomes following damage to prefrontal cortex. Cereb Cortex 6:215-225.

Chambers RA, Taylor JR, Potenza MN (2003) Developmental neurocircuitry of motivation in adolescence: a critical period of addiction vulnerability. Am J Psychiatry 160:1041-1052.

Clark L, Manes F, Antoun N, Sahakian BJ, Robbins TW (2003) The contributions of lesion laterality and lesion volume to decision-making impairment following frontal lobe damage. Neuropsychologia 41:1474-1483.

Critchley HD, Elliot R, Mathias CJ, Dolan RJ (2000) Neural activity relating to generation and representation of galvanic skin conductance responses: a functional magnetic resonance imaging study. J Neurosci 20:3033-3040.

Damasio AR (1996) The somatic marker hypothesis and the possible functions of the prefrontal cortex. Proc Trans R Soc Lond B Biol Sci 351:1413-1420.

Davidson RJ (2004) What the prefrontal cortex "do" in affect: perspectives on frontal EEG asymmetry research. Biol Psychol 67:219-233.

Ernst M, Bolla K, Mouratidis M, Contoreggi C, Matochik JA, Kurian V, Cadet JL, Kimes AS, London ED (2002) Decision-making in a risk-taking task: a PET study. Neuropsychopharmacology 26:682-691.

Fishbein DH, Eldreth DL, Hyde C, Matochik JA, London ED, Contoreggi C, Kurian V, Kimes AS, Breeden A, Grant S (2005) Risky decision making and the anterior cingulate cortex in abstinent drug abusers and nonusers. Cognit Brain Res 23:119-136.

Garavan H, Ross TJ, Stein EA (1999) Right hemispheric dominance of inhibitory control: an event-related functional MRI study. Proc Natl Acad Sci USA 96:8301-8306.

Gershon AA, Dannon PN, Grunhaus L (2003) Transcranial magnetic stimulation in the treatment of depression. Am J Psychiatry 160:835-845.

Ghashghaei HT, Barbas H (2002) Pathways for emotion: interactions of prefrontal and anterior temporal pathways in the amygdala of the rhesus monkey. Neuroscience 115:1261-1279.

Howell DC (1997) Statistical methods for psychology. Belmont, CA: Duxbury.

Johnson E, Tversky A (1983) Affect, generalization, and the perception of risk. J Pers Soc Psychol 36:20-31.

Knoch D, Brugger P, Regard M (2005) Suppressing vs. releasing a habit: frequency-dependent effects of prefrontal transcranial magnetic stimulation. Cereb Cortex 15:885-887.

Knoch D, Treyer V, Regard M, Müri R, Buck A, Weber B (2006) Lateralized and frequency dependent effects of prefrontal rTMS on regional cerebral blood flow. NeuroImage 31:641-648.

Li X, Nahas Z, Kozel FA, Anderson B, Bohning DE, George MS (2004) Acute left prefrontal transcranial magnetic stimulation in depressed patients is associated with immediately increased activity in prefrontal cortical as well as subcortical regions. Biol Psychiatry 55:882-890.

Lieberman MD, Eisenberger N (2004) Conflict and habit: a social cognitive neuroscience approach to the self. In: On building, defending and regulating the self: a psychological perspective (Tesser A, Wood JV, Stapel DA, eds), pp 77-102. New York: Psychology Press.

Manes F, Sahakian B, Clark L, Rogers R, Antoun N, Aitken M, Robbins T (2002) Decision-making processes following damage to the prefrontal cortex. Brain 125:624-639.

Metcalfe J, Mischel WA (1999) Hot/cool-system analysis of delay gratification: dynamics of willpower. Psychol Rev 106:3-19.

Rahman S, Sahakian BJ, Cardinal RN, Rogers RD, Robbins TW (2001) Decision making and neuropsychiatry. Trends Cogn Sci 5:271-277.

Robertson EM, Theoret H, Pascual-Leone A (2003) Studies in cognition: the problems solved and created by transcranial magnetic stimulation. J Cogn Neurosci 15:948-960.

Rogers RD, Owen AM, Middleton HC, Williams EJ, Pickard JD, Sahakian BJ, Robbins TW (1999) Choosing between small, likely rewards and large, unlikely rewards activates inferior and orbital prefrontal cortex. J Neurosci 19:9029-9038.

Starkstein SE, Robinson RG (1997) Mechanisms of disinhibition after brain lesion. J Nerv Ment Dis 185:108-114.

Tranel D, Bechara A, Denburg NL (2002) Asymmetric functional roles of right and left ventromedial prefrontal cortices in social conduct, decisionmaking, and emotional processing. Cortex 38:589-612.

Wassermann EM (1998) Risk and safety of repetitive transcranial magnetic stimulation. Electroencephalogr Clin Neurophysiol 108:1-16. 